\title{
Mehr Wahl, mehr Qual? Zum Zusammenhang von Wahlbeteiligung und neuem Wahlrecht in Hamburg
}

\author{
Michael Jankowski, Cord Jakobeit, Philipp Hiller und Nils Thomsen
}

Das neue Wahlrecht sorgte nicht nur vor, sondern auch nach der Wahl zur Hamburgischen Bürgerschaft am 20. Februar 2011 für ausgiebige Diskussionen. Aus dem wissenschaftlichen Bereich formulierte zuletzt Patrick Horst in der ZParl massive Kritik am neuen Wahlrecht, indem er es als „kontraproduktiv“ bezeichnete und sogar die parlamentarische Demokratie in Hamburg hierdurch als geschädigt ansah. ${ }^{1}$ Sowohl in der Wissenschaft als auch in der Politik wird das neue Wahlrecht vor allem für die um 6,2 Prozentpunkte gesunkene Wahlbeteiligung verantwortlich gemacht. Dieser Rückgang wird insbesondere auf die Komplexität des neuen Wahlrechts zurückgeführt, weshalb eine Vielzahl von Bürgern ${ }^{2}$ entweder ungültig oder gar nicht gewählt hätte.

Um Belege für den von Kritikern aufgestellten Zusammenhang von Wahlrecht und Wahlbeteiligungsrückgang in Hamburg zu finden ${ }^{3}$, wurden Interviews mit knapp 3.000 Wählern und 500 Nichtwählern ausgewertet, die am Wahltag durch die Universität Hamburg im Auftrag der Hamburgischen Bürgerschaft zur Evaluation des neuen Hamburger Wahlrechts durchgeführt wurden. ${ }^{4}$ Dafür werden zum einen die Bewertung des neuen Wahlrechts durch die Wähler und Nichtwähler verglichen. Zum anderen wird anhand der Zustimmung zu Alternativhypothesen geprüft, ob andere Begründungen zum Rückgang der Wahlbeteiligung eine höhere Zustimmung unter den Nichtwählern erhalten haben. Ziel ist es daher nicht, das Nichtwahlverhalten bei der Hamburger Bürgerschaftswahl 2011 vollständig zu erklären, sondern vielmehr Hinweise für oder gegen den behaupteten Zusammenhang einer sinkenden Wahlbeteiligung und einer steigenden Komplexität des Wahlrechts zu finden.

\section{Das neue Hamburger Wahlrecht: Mehr Wahlmöglichkeiten und gesteigerte Komplexität}

\subsection{Zur Geschichte und Funktion des neuen Wahlrechts}

Das neue Hamburger Wahlrecht bildet den Endpunkt eines langjährigen Diskussions- und Verhandlungsprozesses, der maßgeblich zwischen Parteien und der Initiative „Mehr Demokratie“ geführt wurde. Bei der Wahl 2004 bestand für die Wähler in der Hansestadt nur die

1 Patrick Horst, Das neue Hamburger Wahlrecht auf dem Prüfstand, in: ZParl, 42. Jg. (2011), H. 4, S. $707-724$.

2 Um eine bessere Lesbarkeit zu gewährleisten, werden im Rahmen dieses Artikels Doppelungen wie „Bürgerinnen und Bürger“ zugunsten des generischen Begriffs „Bürger“ aufgegeben. Hierdurch soll keine Ungleichbehandlung der Geschlechter signalisiert werden.

3 Wir beschränken uns auf diesen Aspekt, da er uns als der schwerwiegendste Vorwurf am neuen Wahlrecht erscheint.

4 Für den ausführlichen Bericht siehe Cord Jakobeit / Philipp Hiller / Nils Thomsen / Michael Jankowski / Max Pritsch / Daniel Jäckel, Evaluation des neuen Hamburger Wahlrechts. Ergebnisse einer Wähler- und Nichtwählerbefragung, Hamburg, 2011. Auch abrufbar über die Homepage der Hamburgischen Bürgerschaft: http://hamburgische-buergerschaft.de/cms_de. php?templ=presse_detail.tpl\&sub1 $=62 \&$ sub2 $=443 \&$ sub3 $=\&$ cont $=4603$ (Abruf am 7. Juli 2012). 
Möglichkeit, eine von den Parteien vorgegebene Landesliste zu wählen. Durch den erfolgreichen Volksentscheid vom 13. Juni 2004 fand jedoch eine umfassende Reform des Hamburger Wahlrechts statt, das jedoch bereits vor seiner erstmaligen Anwendung erneut durch die damalige CDU-Alleinregierung in Teilen reformiert wurde. Das so reformierte Wahlrecht führte neben der Landesliste auch Wahlkreise ein und bot auf der Wahlkreisliste auch erstmals die Möglichkeit, fünf Stimmen zu panaschieren (das Verteilen der Stimmen auf mehrere Parteilisten oder Kandidaten) oder zu kumulieren (das Anhäufen der Stimmen auf eine einzelne Parteiliste oder Kandidaten). ${ }^{5}$

Nach der Wahl 2008 startete die Initiative „Mehr Demokratie“ erneut ein Volksbegehren zur Reform des Wahlrechts. Zur endgültigen Abstimmung kam dieses Begehren jedoch nicht, da sich CDU, SPD und GAL mit der Initiative auf einen Kompromissvorschlag einigen konnten. ${ }^{6}$ Damit wurde das Wahlrecht von 2008 zur Bürgerschaftswahl 2011 in seiner Komplexität nochmals gesteigert. ${ }^{7}$ Es basiert erneut auf einer Wahlkreis- und einer Landesliste. Auf beiden Listen stehen dem Wähler jeweils fünf Stimmen zur Verfügung, die panaschiert oder kumuliert werden können. Auf der Wahlkreisliste kann keine Partei gewählt werden, sondern nur einzelne Kandidaten. Über den Einzug in die Bürgerschaft entscheidet hierbei allein die Anzahl der erhaltenen Stimmen. ${ }^{8}$ Auf der Landesliste konnten pro Partei bis zu 60 Personen kandidieren, die als einzelne gewählt werden konnten, wie hier jedoch auch die Möglichkeit erhalten blieb, die Parteiliste zu wählen. Das Verhältnis aus Stimmen für die Parteiliste und Stimmen für einzelne Kandidaten bestimmte je nach Partei darüber, bis zu welchem Listenplatz die Kandidaten nach Listenreihenfolge einzogen. Die restlichen Landeslistenplätze wurden erneut nach der Anzahl der meisten Kandidatenstimmen vergeben.

Insgesamt zeigt sich, dass die Wahlmöglichkeiten sehr umfassend sind. Mit insgesamt zehn Stimmen konnten die Wähler über die Zusammensetzung der Bürgerschaft entscheiden. Dieser hohen Stimmenanzahl stand dabei ein noch deutlich größeres Angebot von möglichen Wahlvorschlägen der Parteien gegenüber, so dass sich das neue Wahlrecht als vergleichsweise komplex bezeichnen lässt. Gleichzeitig sollten aber auch nicht die mit dieser Komplexität einhergehenden Gestaltungsmöglichkeiten für die Wähler vernachlässigt werden. Durch die Möglichkeit, die Stimmen gezielt an Kandidaten abzugeben, kann die Zusammensetzung der Bürgerschaft direkt mit beeinflusst werden, wodurch eine bessere

5 Eine umfassende Analyse der Einführung des Hamburger Wahlrechts von 2008 liefert Frank Decker, Parlamentarische Demokratie versus Volksgesetzgebung. Der Streit um ein neues Wahlrecht in Hamburg, in: ZParl, 38. Jg. (2007), H. 1, S. 118 - 133.

6 Hamburger Abendblatt, Kompromiss: Einigung im Wahlrechtsstreit, http://www.abendblatt.de/ hamburg/kommunales/article1034196/Kompromiss-Einigung-im-Wahlrechts-Streit.html (Abruf am 7. Juli 2012). Ein ausführlicher Überblick über die Einführung des neuen Wahlrechts 2011 findet sich außerdem bei Klaus David, Wechselfälle des Wahlrechts in Hamburg. Oder: Die Verhinderung eines weiteren Volksentscheids über das Wahlrecht, in: ZParl, 41. Jg. (2010), H. 3, S. $598-622$.

7 Wir konzentrieren uns hier auf die wichtigsten Änderungen. Eine ausführliche Erklärung des Wahlrechts liefert die Broschüre von Frederike David / Klaus David, 20 Stimmen für Hamburg. Das neue Wahlrecht zur Hamburgischen Bürgerschaft und zu den Bezirksversammlungen, Hamburg 2011.

8 Bei der Wahl 2008 gab es in den Wahlkreisen noch ein Quorum an Stimmen, das erreicht werden musste, um die von der Partei aufgestellte Listenreihenfolge zu verändern. 
Berücksichtigung des Wählerwillens gegeben ist. Die Komplexität allein kann daher nicht Ziel der Kritik sein, da sich die hiermit einhergehenden Änderungen zumindest theoretisch als Gewinn für die Demokratie verstehen lassen.

\subsection{Die Entwicklung der Wahlbeteiligung in Hamburg}

Die Debatte um den Effekt des neuen Wahlrechts auf die Wahlbeteiligung ist von so großer Relevanz, weil bei der Wahl 2011 die Beteiligung um 6,2 Prozentpunkte gegenüber 2008 auf 57,3 Prozent sank. Damit verschärfte sich auch in Hamburg ein seit Jahren auf allen Ebenen des politischen Systems beobachtbarer Rückgang der Wahlbeteiligung, der sich in nahezu allen westlichen Demokratien feststellen lässt. ${ }^{9}$ Schon die Beteiligung von 63,5 Prozent im Jahr 2008 markierte einen historischen Tiefpunkt in Hamburg. Der Einbruch von 2011 ist jedoch trotz dieses niedrigen Ausgangsniveaus der stärkste seit der Wahl von 1991, als die Beteiligung von 79,5 (1987) auf 66,1 Prozent gesunken war und in den Folgejahren bis 2001 wieder auf 71 Prozent anstieg (siehe Abbildung 1).

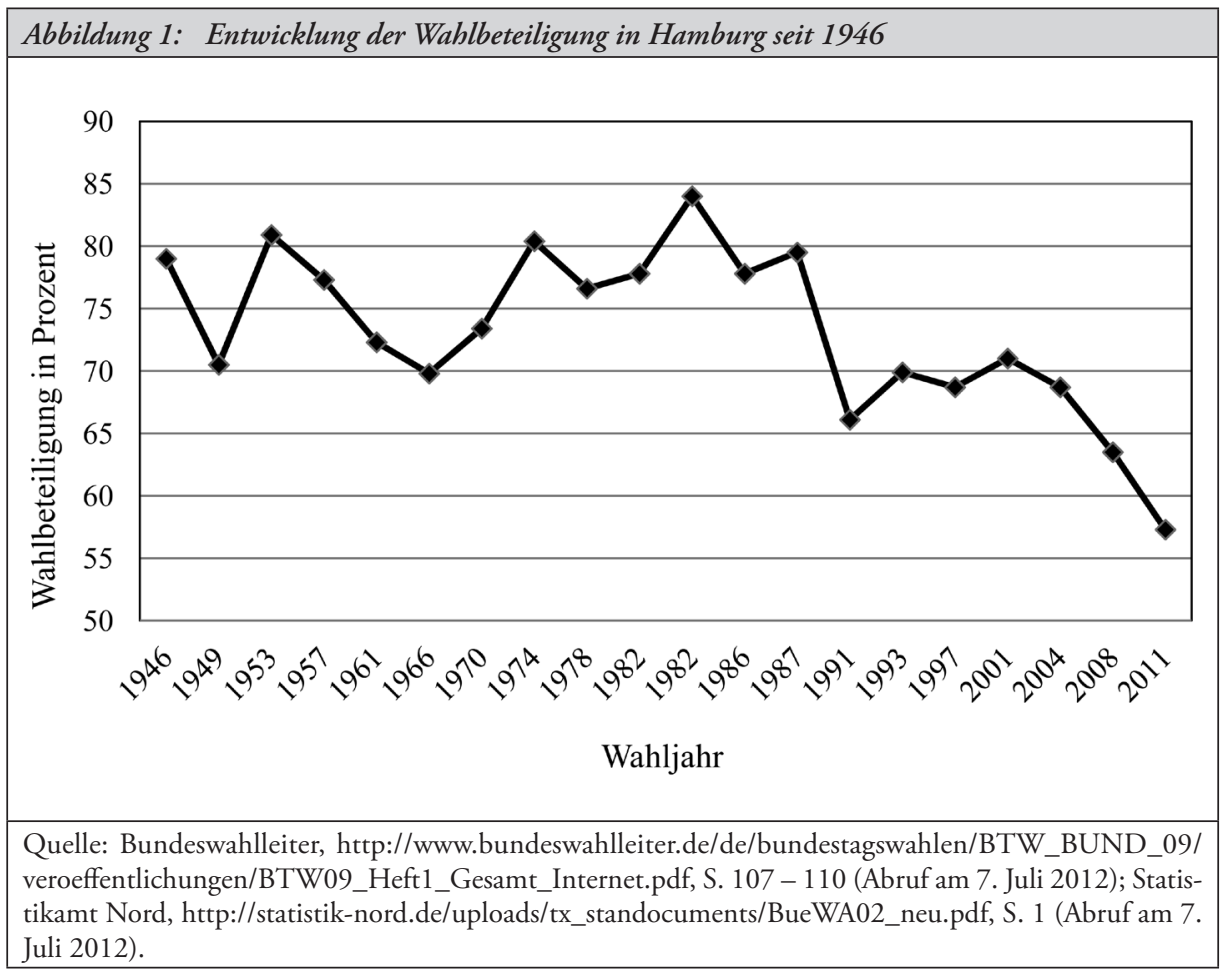

9 Für einen umfassenden internationalen Vergleich von Wahlbeteiligungen und die Diskussion der Effekte von Nichtwählern für die Demokratie siehe Dorothée de Nève, Die NichtwählerInnen. Eine Gefahr für die Demokratie?, Opladen 2009. 


\subsection{Das neue Wahlrecht: Ursache für den Rückgang der Wahlbeteiligung?}

Nach Ansicht der Kritiker des neuen Wahlrechts führte insbesondere die Komplexität zu dem beschriebenen Rückgang der Beteiligung an der Bürgerschaftswahl 2011. So stellt Patrick Horst basierend auf der Studie, auf deren Daten auch dieser Artikel basiert, fest, dass 53 Prozent der Nichtwähler das neue Wahlrecht als zu kompliziert empfanden. ${ }^{10}$ Er schlussfolgert: „Das neue Wahlrecht bewirkte also durchaus entgegen seiner Absicht ein Sinken der Wahlbeteiligung. " ${ }^{11}$ Zusammenfassend stellt er dem neuen Wahlrecht daher ein negatives Urteil aus und sieht hierdurch gar eine Gefahr für die parlamentarische Demokratie: „Die Bürger waren mit der Komplexität und dem überbordenden, aber gänzlich unbekannten Personalangebot der Parteien überfordert, weshalb sie öfter als in der Vergangenheit ungültig stimmten oder der Wahl fernblieben. Die Legitimation und Akzeptanz der parlamentarischen Demokratie nahm weiteren Schaden. "12 Diese Einschätzung teilt Patrick Horst mit Vertretern der Politik. Ähnlich urteilte etwa die jetzige Wissenschaftssenatorin Dorothee Stapelfeld (SPD), die erklärte, die gesunkene Wahlbeteiligung „muss für uns alle parteiübergreifend ein Alarmsignal sein. Es kann uns nicht ruhig lassen, wenn Menschen sich bewusst abwenden und sich gegen diese Teilhabemöglichkeiten entscheiden. Es kann uns ebenso wenig ruhig lassen, wenn sie aus Furcht, den Stimmzettel nicht zu verstehen, fernbleiben und auf die Wahrnehmung ihres Wahlrechts verzichten"13.

Zusammenfassend zeigt sich bei den hier angesprochenen Kritikern eine direkte Gleichsetzung der Komplexität des neuen Wahlrechts mit der sinkenden Wahlbeteiligung. Jedoch erfolgt diese Gleichsetzung weitestgehend ohne eine empirische Grundlage. Auch der Verweis von Patrick Horst auf unsere Evaluation des Wahlrechts erachten wir als zu einseitig und plädieren dafür, diese im Rahmen der gesamten Evaluationsergebnisse zu bewerten.

\section{Erklärungen für Nichtwahlverhalten}

\subsection{Erkenntnisse der Nichtwählerforschung}

Nichtwähler wurden lange Zeit in der Wahlforschung vernachlässigt. In den meist unzähligen Analysen zum Ausgang einer Wahl gilt der Höhe der Wahlbeteiligung in der Regel höchstens eine Randbemerkung. Nichtwahlverhalten wurde bislang nur selten umfassend und systematisch untersucht. ${ }^{14}$ Hervorzuheben sind die Dissertationen von Michael Eilfort und Thomas Kleinhenz aus den neunziger Jahren. ${ }^{15}$ Zuletzt veröffentlichte Dorothée de Nève

10 Vgl. Patrick Horst, a.a.O. (Fn. 1), S. 721.

11 Vgl. ebenda, S. 721.

12 Ebenda, S. $722 \mathrm{f}$.

13 Plenarprotokoll 20/1 der Bürgerschaft der Freien und Hansestadt Hamburg, vom 7. März 2011, S. 8.

14 Erste Erkenntnisse in einer vergleichenden Studie bei Leonhard Neidhart / Jean-Pierre Hoby, Ursachen der gegenwärtigen Stimmabstinenz in der Schweiz: ein Forschungsbericht im Auftrag der Justizabteilung des Eidgenössischen Justiz- und Polizeidepartementes, Zürich 1977.

15 Vgl. Michael Eilfort, Die Nichtwähler: Wahlenthaltung als Form des Wahlverhaltens, Paderborn 1994; Thomas Kleinhenz, Die Nichtwähler: Ursachen der sinkenden Wahlbeteiligung in Deutschland, Opladen 1995. 
eine umfassende Analyse, in der Nichtwähler einem internationalen Vergleich unterzogen werden. ${ }^{16}$ Daneben mehren sich aber seit den neunziger Jahren auch wissenschaftliche Artikel zum Nichtwahlverhalten. Insgesamt liegt daher trotz vergleichsweise geringer Forschung eine Reihe zentraler Erkenntnisse zur Erklärung von Nichtwahlverhalten vor.

Grundsätzlich erweisen sich ein hohes politisches Interesse und das Vorhandensein einer Parteiidentifikation als wichtige Erklärungsfaktoren für das Wahlverhalten. ${ }^{17}$ Des Weiteren besteht ein starker Zusammenhang zwischen sozioökonomischen Ressourcen und der Beteiligung an Wahlen. ${ }^{18}$ Kombiniert man diese beiden Erkenntnisse, ergibt sich das Bild eines politisch desinteressierten und sozial schwachen Nichtwählers. Wenngleich diese Typbeschreibung sicherlich auf eine Reihe von Nichtwählern zutrifft, ist insbesondere in der jüngeren Literatur dieses Bild kritisiert worden. So wird zunehmend auch ein „Nichtwähler neuen Typs" diskutiert, der sich gerade durch eine höhere Bildung und politisches Interesse auszeichnet. Dieser möchte durch seine Nichtwahl Protest ausdrücken, und zwar in dem Sinne, dass er mehr und neue Beteiligungsformen sucht. ${ }^{19}$

Obwohl diese Erkenntnisse wichtig für die Nichtwählerforschung sind, können sie für die vorliegende Analyse nicht direkt nutzbar gemacht werden. Die herangezogenen Faktoren erklären letztlich unabhängig von der spezifischen Wahl, weshalb Personen nicht wählen gehen. Hier sind jedoch Erklärungen für den Rückgang der Wahlbeteiligung nötig, wofür wahlspezifische Erklärungen interessant sind - also solche, die die politische Situation einer Wahl berücksichtigen. ${ }^{20}$ Die von den Kritikern aufgestellte „Wahlrechtshypothese“ ist eine solche Erklärung. Aus der Nichtwählerforschung lassen sich aber noch zwei weitere ableiten, die ebenso auf die Hamburger Bürgerschaftswahl angewendet werden können.

\subsection{Wahlspezifische Erklärungen für den Rückgang der Wahlbeteiligung}

Die erste wahlspezifische Erklärung für die Höhe der Wahlbeteiligung ist die vermutete Knappheit des Wahlausgangs. Das Argument lässt sich aus der Rational-Choice-Theorie ableiten, nach der die Beteiligung an Wahlen auf Kosten-Nutzen-Überlegungen des Wählers

16 Vgl. Dorothée de Nève, a.a.O. (Fn. 9).

17 Siehe beispielsweise Kerstin Völkl, Nichtwahl - die Alternative für politisch ungebundene am Wahltag?, in: Hans Rattinger / Oscar W. Gabriel / Jürgen W. Falter (Hrsg.), Der gesamtdeutsche Wähler, Baden-Baden 2007, S. 15 - 36 oder Klaus Armingeon, Gründe und Folgen geringer Wahlbeteiligung, in: Kölner Zeitschrift für Soziologie und Sozialpsychologie, 46. Jg. (1994), H. 1, S. $43-64$.

18 Vgl. beispielsweise Alexander Gattig, Klasseneinflüsse auf das Wahlverhalten und die Wahlbeteiligung. Resultate aus der Bundesrepublik Deutschland und den USA, in: Kölner Zeitschrift für Soziologie und Sozialpsychologie, 58. Jg. (2006), H. 3, S. $510-533$.

19 Für eine kritische Diskussion dieses Nichtwählertyps siehe Oscar W. Gabriel / Kerstin Völkl, Auf der Suche nach dem Nichtwähler neuen Typs. Eine Analyse aus Anlass der Bundestagswahl 2002, in: Frank Brettschneider / Jan van Deth / Edeltraud Roller (Hrsg.), Die Bundestagswahl 2002. Analysen der Wahlergebnisse und des Wahlkampfs, Wiesbaden 2004, S. 221 - 248.

20 Natürlich könnte der Rückgang der Wahlbeteiligung auch daran liegen, dass es eine Veränderung in den Variablen gab, die grundsätzlich zur Erklärung von Nichtwahlverhalten dienen. Beispielsweise könnte sich der Anteil politisch desinteressierter Personen erhöht haben. Dieser Aspekt kann hier nicht berücksichtigt werden. Die Annahme, dass sich diese Variablen nicht verändert haben, teilen wir aber auch mit den Kritikern des neuen Wahlrechts, die implizit die selbe Grundannahme treffen. 
basiert. ${ }^{21}$ Wenn der Wahlausgang schon vor der Wahl als quasi entschieden gilt, dann sinkt der individuelle Nutzen der Teilnahme, da die eigene Stimme kaum einen nennenswerten Effekt haben wird. Entsprechend steigt die Wahlbeteiligung, wenn Umfragen ein Kopf-anKopf-Rennen vorhersagen. ${ }^{22}$ Diese Hypothese wird daher im Folgenden als „RationalChoice-Hypothese" bezeichnet.

Die zweite wahlspezifische Erklärung leitet sich aus der so genannten Michigan-School der Wahlforschung ab. Dieser sozialpsychologische Ansatz basiert auf dem Zusammenspiel zwischen einer langfristigen, relativ stabilen Parteiidentifikation und zwei kurzfristigen, wahlspezifischen Faktoren ${ }^{23}$, der Kandidaten- und der Themenpräferenz. Mit ersterer ist die Sympathie für den jeweiligen Spitzenkandidaten gemeint. Die Themenpräferenz zielt insbesondere auf Gegenstände ab, die vor der Wahl besonders stark diskutiert wurden. Stimmen die Parteiidentifikation, die Kandidaten- und Themenpräferenz überein, erfolgt in der Regel die Wahl der Partei, der man sich im Sinne der Parteiidentifikation zugehörig fühlt. Widersprechen sich jedoch Parteiidentifikation und Kandidaten-/Themenpräferenz oder liegt keine Kandidaten- oder Themenpräferenz vor, kann es zu einer Wahlenthaltung kommen. ${ }^{24}$ Da sich die beiden kurzfristigen Präferenzen maßgeblich auf Aspekte des Wahlkampfs beziehen, wird diese zweite Hypothese als „Wahlkampfhypothese“ bezeichnet.

Bezieht man die „Rational-Choice-Hypothese“ und die „Wahlkampfhypothese“ auf die Hamburger Bürgerschaftswahl, erscheinen beide plausibel. Für erstere spricht, dass die Umfragen vor der Bürgerschaftswahl schon lange einen klaren Sieg der SPD vorausgesagt hatten. ${ }^{25}$ Fraglich war hier letztlich nur, ob es zu einer Alleinregierung reichen oder ob die SPD auf die GAL als Koalitionspartner angewiesen sein würde - ein Aspekt der als zweitrangig gelten kann, da Rot-Grün gemeinhin als ein Lager wahrgenommen wird.

Für die „Wahlkampfhypothese“ spricht ein insgesamt unausgewogenes Personalangebot der Parteien. Für die SPD kandidierte mit Olaf Scholz ein prominenter Bundespolitiker für das Amt des Bürgermeisters. Das Personalangebot der anderen Parteien war dagegen deutlich weniger prominent und hatte keine so hohe Beliebtheit unter den Bürgern. Insbesondere der damalige Bürgermeister und Spitzenkandidat der CDU, Christoph Ahlhaus, hatte mit vergleichsweise niedrigen Bekanntheits- und Beliebtheitswerten zu kämpfen. ${ }^{26}$ Hinzu

21 Vgl. hierzu allgemein Kai Arzheimer / Annette Schmitt, Der ökonomische Ansatz, in: Jürgen W. Falter / Harald Schoen (Hrsg.), Handbuch Wahlforschung, Wiesbaden 2005, S. 317 - 336.

22 Empirisch bestätigt wurde dieser Zusammenhang unter anderem von Jürgen W. Falter / Siegfried Schumann, Der Nichtwähler - das unbekannte Wesen, in: Hans-Dieter Klingemann / Max Kaase (Hrsg.), Wahlen und Wähler. Analysen aus Anlass der Bundestagswahl, Opladen 1990, S. 183 184.

23 Vgl. hierzu allgemein Harald Schoen / Cornelia Weins, Der sozialpsychologische Ansatz zur Erklärung von Wahlverhalten, in: Jürgen W. Falter / Harald Schoen (Hrsg.), a.a.O. (Fn. 21), S. 187 241.

24 So argumentiert beispielsweise auch Claudio Caballero, Nichtwahl, in: Jürgen W. Falter / Harald Schoen (Hrsg.), a.a.O. (Fn. 21), S. 329 - 365, S. 347.

25 Hamburger Abendblatt, Hamburgs SPD in Wahlumfrage fast doppelt so stark wie die CDU, http://www.abendblatt.de/hamburg/kommunales/article1785094/Hamburgs-SPD-in-Wahlumfrage-fast-doppelt-so-stark-wie-die-CDU.html (Abruf am 7. Juli 2012).

26 Die Beliebtheit von Christoph Ahlhaus lag auf einer Höhe mit der von GAL-Spitzenkandidatin Anja Hajduk, während die Beliebtheitswerte von Olaf Scholz doppelt so hoch waren. Vgl. hierzu Spiegel Online, Rot-Grün liegt in Hamburg deutlich vorn, http://www.spiegel.de/politik/deutschland/umfrage-rot-gruen-liegt-in-hamburg-deutlich-vorn-a-739364.html (Abruf am 7. Juli 2012). 
kommt, dass auch der Wahlkampf allgemein als ruhig wahrgenommen wurde und keine offensive Diskussion bestimmter Themen stattfand. Es erscheint daher plausibel, dass das Personalangebot und der müde Wahlkampf bei manchen Bürgern nicht zu einer Politisierung führten und sie sich daher entschlossen, der Wahl fernzubleiben. Mit anderen Worten: Eine Kandidaten- und Themenpräferenz konnte sich im Wahlvorfeld nur schwer entwickeln und setzte eine intensive Beschäftigung mit der Politik voraus, die nicht für alle Bürger angenommen werden kann.

Beide Hypothesen liefern somit nicht nur theoretisch weitere Erklärungen für die sinkende Wahlbeteiligung, sondern erweisen sich gerade aufgrund der damaligen politischen Situation in Hamburg als geeignet. Sie treten in Konkurrenz zu der Hypothese, dass das Wahlrecht die niedrigere Wahlbeteiligung verursacht hat. Welche empirischen Befunde lassen sich für diese drei Hypothesen bei den Hamburger Wählern und Nichtwählern finden?

\section{Analyse der Hamburger Wähler und Nichtwähler}

\subsection{Das Vorgehen bei der Wähler- und Nichtwählerbefragung in Hamburg 27}

Die Befragung der Hamburger Wähler und Nichtwähler erfolgte im Auftrag der Hamburgischen Bürgerschaft durch die Universität Hamburg zum Zwecke der Evaluation des neuen Wahlrechts. 3.104 Wähler wurden in allen Hamburger Stadtteilen in zufällig ausgewählten Wahllokalen direkt nach ihrer Stimmabgabe befragt. Im Fokus standen dabei Fragen zur Bewertung des neuen Wahlrechts und zum Wahlverhalten.

Die Befragung von Nichtwählern stellt allgemein eine methodische Herausforderung dar. Vorteil und Nachteil zugleich war die Tatsache, dass die Nichtwähler am Wahltag selbst befragt wurden. Der Vorteil besteht darin, dass die gängige Unterscheidung in bekennende und nicht-bekennende Nichtwähler nicht erfolgen musste. Diese Unterscheidung wird von Studien meist eingeführt, um solche Befragte, die eine Nichtwahlabsicht nicht direkt zugeben, jedoch hinsichtlich ihrer Wahlabsicht mit „weiß nicht“ antworten, auch als mögliche Nichtwähler in die Analyse einzubeziehen. ${ }^{28}$ Hier kann die wirkliche Wahlenthaltung letztlich nur vermutet werden. Durch die Befragung der Nichtwähler am Wahltag entfiel die Möglichkeit der „,weiß nicht“-Antwort, wodurch weitestgehend sichergestellt werden konnte, dass nur (bekennende) Nichtwähler befragt wurden. Nachteil war hingegen die Schwierigkeit, an einem einzigen Tag überhaupt genügend bekennende, zum Interview bereite Nichtwähler zu finden. Die Befragungen wurden an über dreißig verschiedenen öffentlichen Plätzen in unterschiedlichen Stadtteilen durchgeführt. Letztlich konnten über dieses Vorgehen 494 Nichtwähler befragt werden. ${ }^{29}$

27 Ausführlich ist das Vorgehen in der Evaluation des neuen Hamburger Wahlrechts dargelegt in: Cord Jakobeit / Philipp Hiller / Nils Thomsen / Michael Jankowski / Max Pritsch / Daniel Jäckel, a.a.O. (Fn. 4), S. $85-93$.

28 Siehe beispielsweise Jürgen W. Falter / Siegfried Schumann, a.a.O. (Fn. 21), S. 175.

29 Aufgrund der unterschiedlichen Responsivität bei manchen Fragen schwankt die Fallzahl in den nachfolgenden Tabellen zwischen 397 und 478 Nichtwählern. 


\subsection{Einstellungen der Nichtwähler zur Komplexität des Wahlrechts}

Zunächst wurden die Hamburger Nichtwähler nach ihrer Einschätzung des neuen Wahlrechts befragt. Das neue Wahlrecht wurde zwar von etwas über der Hälfte als zu kompliziert wahrgenommen, gleichzeitig aber auch von der Mehrheit der Nichtwähler als Stärkung der Wählermacht verstanden. Die geringe Zustimmung zu Aussagen wie „Es wirkt der Politikverdrossenheit entgegen “ und „Es schafft eine größere Nähe von Wählern und Kandidaten“, zeigt jedoch, dass das Wahlrecht von den Nichtwählern mehrheitlich kritisch betrachtet wird. Nach ihrer Auffassung wird es den gesetzten Zielen wie Minderung der Politikverdrossenheit und Einschränkung des Parteieneinflusses nicht gerecht (vgl. Tabelle 1).

\begin{tabular}{|l|c|c|c|c|}
\hline Tabelle 1: Zustimmung zu Aussagen über das neue Wahlrecht (in Prozent) \\
\hline Aussage & $\begin{array}{c}\text { Stimme voll } \\
\mathrm{zu}\end{array}$ & $\begin{array}{c}\text { Stimme eher } \\
\mathrm{zu}\end{array}$ & $\begin{array}{c}\text { Stimme eher } \\
\text { nicht zu }\end{array}$ & $\begin{array}{c}\text { Stimme gar } \\
\text { nicht zu }\end{array}$ \\
\hline Es ist zu wenig erläutert worden. (N = 406) & 33,5 & 25,4 & 21,7 & 19,5 \\
Es stärkt die Macht der Wähler. (N = 397) & 22,4 & 31,2 & 23,7 & 22,7 \\
Es ist zu kompliziert. (N = 423) & 32,6 & 20,1 & 23,6 & 23,6 \\
Es schafft eine größere Nähe von Wählern & 18,3 & 25,4 & 30,9 & 25,4 \\
und Kandidaten. (N = 405) & & & & 29,7 \\
Es wirkt der Politikverdrossenheit entgegen. & 17,2 & 19,9 & 33,2 \\
(N = 401) & & & \\
\hline Quelle: Eigene Erhebung im Rahmen der Evaluation des neuen Hamburger Wahlrechts, vgl. Cord Jakobeit \\
/ Philipp Hiller / Nils Thomsen / Michael Jankowski / Max Pritsch / Daniel Jäckel, a.a.O. (Fn. 4). \\
\hline
\end{tabular}

Interessant ist, dass das neue Wahlrecht trotz seiner Komplexität von den Nichtwählern nicht als sonderlich schlechter als das sehr einfache Wahlrecht von 2004 mit nur einer Stimme bewertet wird. Zwar empfinden nur 5,3 Prozent das Wahlrecht als „sehr viel besser“. Jedoch sagen 37,8 Prozent der Nichtwähler, das neue Wahlrecht sei „eher besser“, und 28 Prozent schätzen es zumindest als „gleich gut/schlecht“ ein im Vergleich zu 2004. Nur eine Minderheit von insgesamt 28,9 Prozent bezeichnet das neue Wahlrecht als schlechter (18,1 Prozent eher schlechter und 10,8 Prozent sehr viel schlechter). Es schneidet in der Bewertung zwar als zu kompliziert ab; diese Einschätzung führt aber nicht dazu, dass die Mehrheit der Nichtwähler es verglichen zu einem deutlich einfacheren Wahlrecht mehrheitlich ablehnt.

\subsection{Vergleich der Einschätzung des neuen Wahlrechts zwischen Nichtwählern und Wählern}

Hiermit ist jedoch noch nicht gezeigt, inwiefern das komplexe Wahlrecht der entscheidende Grund für das Sinken der Wahlbeteiligung war. Ein erster Hinweis lässt sich finden, wenn man die Einschätzung der Komplexität und die Bewertung des Wahlrechts durch die Nichtwähler mit den Angaben der Wähler zu diesen Fragen vergleicht. Eine deutlich schlechtere Bewertung des neuen Wahlrechts durch die Nichtwähler würde dafür sprechen, dass es einen 
abschreckenden Effekt auf die Bürger gehabt hat. Lassen sich jedoch keine Unterschiede feststellen, würde dies gegen die Wahlrechtsthese sprechen.

Der Vergleich zeigt, dass lediglich graduelle Unterschiede zwischen Wählern und Nichtwählern bestehen. Zwar bewerten mehr Wähler das neue Wahlrecht als „sehr viel besser“ oder „eher besser“ als Nichtwähler (56,2 Prozent Zustimmung bei Wählern, 43,1 Prozent bei Nichtwählern), dennoch entspricht dies bei beiden Gruppen der Gruppenmehrheit. Interessanterweise bewertet mehr als jeder vierte Nichtwähler das neue Wahlrecht als "gleich“ (28,0 Prozent), bei den Wählern gibt dies nur jeder sechste (16,0 Prozent) an. Weitestgehende Übereinstimmung herrscht bei der negativen Bewertung des neuen Wahlrechts. Die Wähler beurteilen es zu 27,8 Prozent als „eher schlecht“ oder „sehr viel schlechter“, während insgesamt 28,9 Prozent der Nichtwähler diese Einschätzung teilen. Somit liegt hier eine große Deckungsgleichheit von Wählern und Nichtwählern vor (vgl. Tabelle 2).

\begin{tabular}{|l|c|c|}
\hline Tabelle 2: $\begin{array}{c}\text { Bewertung des neuen Wablrechts im Vergleich zum Wablrecht von 2004 (mit nur einer } \\
\text { Stimme) durch die Wähler und Nichtwähler (in Prozent) }\end{array}$ \\
\hline Bewertung & Wähler (N = 2.522) & Nichtwähler $(\mathrm{N}=415)$ \\
\hline sehr viel besser & 10,5 & 5,3 \\
eher besser & 45,7 & 37,8 \\
gleich & 16,0 & 28,0 \\
eher schlechter & 21,0 & 18,1 \\
sehr viel schlechter & 6,8 & 10,8 \\
\hline Quelle: Vgl. Tabelle 1. & \\
\hline
\end{tabular}

Auch ein Vergleich der Einschätzungen der Komplexität des Wahlrechts spricht nicht für die „Wahlrechtshypothese“. Die Wähler stimmen etwas weniger der Aussage zu, dass das neue Wahlrecht zu kompliziert sei, als die Nichtwähler. Der Unterschied beträgt jedoch nur 4,3 Prozentpunkte (52,7 Prozent Zustimmung der Nichtwähler und 48,4 Prozent Zustimmung der Wähler, vgl. Tabelle 3). Aus Sicht der "Wahlrechtshypothese“ wäre hier ein deutlich größerer Unterschied in der Bewertung zu erwarten gewesen.

\begin{tabular}{|l|c|c|}
\hline Tabelle 3: Zustimmung der Wähler und Nichtwähler zu der Aussage „Das neue Wablrecht ist zu \\
kompliziert“ (in Prozent)
\end{tabular}

\subsection{Motive der Hamburger Nichtwähler für die Wahlenthaltung}

Folgt man dem Argument der Kritiker, so müssten gerade diejenigen Befragten, die das neue Wahlrecht als zu kompliziert empfanden, aus diesem Grund der Wahl ferngeblieben sein. 
Um dies zu prüfen, lohnt sich eine Analyse der Nichtwahlmotive dieser Nichtwählergruppe. Hierbei lässt sich feststellen, dass selbst bei dieser Gruppe von Nichtwählern das Wahlrecht nur ein Grund unter vielen für die Wahlenthaltung war. ${ }^{30}$

Wenngleich auch hier die Komplexität des neuen Wahlrechts eine hohe Zustimmung von 75,1 Prozent erfährt, muss es trotzdem überraschen, dass sich dieses Motiv in direkter Konkurrenz zu Aussagen wie „Der Wahlkampf hat mich nicht angesprochen“ (79,9 Prozent) und „Die Kandidaten überzeugen nicht“ (69,1 Prozent) befindet (siehe Tabelle 4). Interessant ist aber insbesondere, dass die meisten Motive der Tabelle 4 den anderen Hypothesen, die zur Erklärung des Wahlbeteiligungsrückgangs aufgestellt wurden, entsprechen. So bestätigen die hohe Zustimmung zu „Der Wahlkampf hat mich nicht angesprochen“ und „Die Kandidaten überzeugen nicht" die "Wahlkampfhypothese“, nach der die Kandidaten und Themen vor einer Wahl die Wahlentscheidung beeinflussen. Auch die „Rational-ChoiceHypothese“, nach der die Vorhersehbarkeit des Wahlausgangs die Höhe der Wahlbeteiligung beeinflusst, findet sich in der Aussage „Die Wahl scheint mir schon entschieden“ $(54,4$ Prozent) wieder.

\begin{tabular}{|c|c|c|c|c|}
\hline \multicolumn{5}{|c|}{$\begin{array}{l}\text { Tabelle 4: Motive der Wablenthaltung bei Hamburger Nichtwählern, die das Wablrecht als zu } \\
\text { kompliziert empfinden (in Prozent) }\end{array}$} \\
\hline Aussage & $\begin{array}{l}\text { Stimme } \\
\text { voll zu }\end{array}$ & $\begin{array}{l}\text { Stimme } \\
\text { eher zu }\end{array}$ & $\begin{array}{c}\text { Stimme eher } \\
\text { nicht } \mathrm{zu}\end{array}$ & $\begin{array}{l}\text { Stimme gar } \\
\text { nicht zu }\end{array}$ \\
\hline $\begin{array}{l}\text { Der Wahlkampf hat mich nicht angesprochen. } \\
(\mathrm{N}=219)\end{array}$ & 59,8 & 20,1 & 11,0 & 9,1 \\
\hline Das Wahlrecht ist zu kompliziert. $(\mathrm{N}=221)$ & 51,6 & 23,5 & 8,6 & 16,3 \\
\hline Die Kandidaten überzeugen nicht. $(\mathrm{N}=214)$ & 42,5 & 26,6 & 15,9 & 15,0 \\
\hline $\begin{array}{l}\text { Eine Wahl alle vier Jahre reicht nicht aus, } \\
\text { es muss mehr Volksentscheide geben. } \\
(\mathrm{N}=220)\end{array}$ & 39,6 & 15,9 & 18,2 & 26,4 \\
\hline $\begin{array}{l}\text { Die Wahl scheint mir schon entschieden. } \\
(\mathrm{N}=223)\end{array}$ & 33,2 & 21,2 & 16,6 & 29,0 \\
\hline $\begin{array}{l}\text { Es gibt keine Partei, die meine Interessen } \\
\text { vertritt. }(\mathrm{N}=215)\end{array}$ & 28,8 & 22,8 & 20,9 & 27,4 \\
\hline
\end{tabular}

Unter Berücksichtigung dieser Zustimmungsraten kann daher festgestellt werden, dass selbst in der Gruppe der Nichtwähler, die das Wahlrecht als zu kompliziert empfinden, dies nur einer von vielen Gründen ist, die zur Nichtwahlentscheidung beigetragen haben.

Betrachtet man nicht nur eine Untergruppe von Nichtwählern, sondern die Motive aller Nichtwähler, nicht an der Wahl teilzunehmen, wird noch deutlicher, dass das neue Wahlrecht nur eine vergleichsweise geringe Bedeutung im Vergleich zu den anderen Hypothesen

30 In Tabelle 4 werden nur Nichtwähler betrachtet, die das neue Wahlrecht allgemein als zu kompliziert bewertet haben. Die Frage nach der Komplexität in der Tabelle 4 fragt jedoch danach, ob das neue Wahlrecht auch das Motiv der Nichtwahlentscheidung war. 
hatte: 77,0 Prozent der Nichtwähler geben an, dass der Wahlkampf sie nicht angesprochen hat, gefolgt von der Aussage „Die Kandidaten überzeugen nicht“ (67,4 Prozent). Neben der Forderung nach mehr Volksentscheiden (54,6 Prozent) findet außerdem die Aussage „Die Wahl scheint mir schon entschieden“ (51,1 Prozent) eine mehrheitliche Zustimmung. Die Komplexität des Wahlrechts als Motiv für die Nichtwahl nennen hingegen nur 48,5 Prozent (vgl. Tabelle 5). Dies spricht erneut dafür, dass die anderen Hypothesen zur Erklärung der Wahlenthaltung eine größere Rolle spielen. Anstelle des Wahlrechts erscheinen insbesondere ein unattraktiver Wahlkampf, ein schwaches Kandidatenangebot und auch das weitgehend absehbare Wahlergebnis wichtigere Motive für die Nichtwahlentscheidung gewesen zu sein.

\begin{tabular}{|c|c|c|c|c|}
\hline Aussage & $\begin{array}{c}\text { Stimme voll } \\
\mathrm{zu}\end{array}$ & $\begin{array}{c}\text { Stimme eher } \\
\mathrm{zu}\end{array}$ & $\begin{array}{c}\text { Stimme eher } \\
\text { nicht zu }\end{array}$ & $\begin{array}{c}\text { Stimme gar } \\
\text { nicht zu }\end{array}$ \\
\hline $\begin{array}{l}\text { Der Wahlkampf hat mich nicht } \\
\text { angesprochen. }(\mathrm{N}=474)\end{array}$ & 58,6 & 18,4 & 12,2 & 10,8 \\
\hline Die Kandidaten überzeugen nicht. $(\mathrm{N}=469)$ & 43,3 & 24,1 & 18,6 & 14,1 \\
\hline $\begin{array}{l}\text { Eine Wahl alle vier Jahre reicht nicht, es muss } \\
\text { mehr Volksentscheide geben. } \\
(\mathrm{N}=478)\end{array}$ & 35,1 & 19,5 & 17,6 & 27,8 \\
\hline $\begin{array}{l}\text { Die Wahl scheint mir schon entschieden. } \\
(\mathrm{N}=473)\end{array}$ & 30,0 & 21,1 & 20,1 & 28,8 \\
\hline $\begin{array}{l}\text { Es gibt keine Partei, die meine Interessen ver- } \\
\text { tritt. }(\mathrm{N}=473)\end{array}$ & 29,4 & 21,4 & 21,8 & 27,5 \\
\hline Das Wahlrecht ist zu kompliziert. $(\mathrm{N}=477)$ & 27,5 & 21,0 & 13,6 & 37,9 \\
\hline
\end{tabular}

\section{Fazit: Kaum eine Reform nötig}

Obwohl also das neue Wahlrecht von vielen Nichtwählern als zu komplex wahrgenommen wurde, scheint dies nicht der Hauptgrund für den Rückgang der Wahlbeteiligung in Hamburg gewesen zu sein. Tatsächlich fällt das Wahlrecht gegenüber anderen wahlspezifischen Faktoren nicht so stark ins Gewicht. Vielmehr dürfte die Wahlbeteiligung durch den schwachen Wahlkampf und die Unzufriedenheit mit dem Kandidatenangebot beeinflusst worden sein. Auch der vorhersehbare Sieg der SPD war für viele Nichtwähler ein wichtiger Grund, nicht zur Wahl zu gehen. Wenngleich sich ein Einfluss des neuen Wahlrechts nicht vollständig ausschließen lässt, scheint dieser jedoch vergleichsweise gering gewesen zu sein. Wäre das Wahlrecht bei einer Wahl eingeführt worden, bei der kein Sieger abzusehen gewesen wäre, ein klarer Streit um Themen stattgefunden hätte und ein ausgeglichenes Spitzenkandidatenangebot vorhanden gewesen wäre, wäre die Beteiligung daher entweder deutlich schwächer oder vielleicht gar nicht zurückgegangen. Folglich können die „neuen Nichtwähler“ nicht als dauerhaft von der Wahlteilnahme abgeschreckt gelten, sondern ihr Verhalten dürfte maßgeblich auf die politischen Umstände zurückzuführen sein. 
Insgesamt lässt die Berücksichtigung dieser Ergebnisse das Hamburger Wahlrecht in einem anderen Licht erscheinen. Zwar kann es nicht zuletzt für eine erhöhte Anzahl von ungültigen Stimmen bei der Bürgerschaftswahl verantwortlich gemacht werden, einen generell abschreckenden Effekt auf die Wähler hatte es aber nicht. Während letzteres in der Tat ein erhebliches Problem für die Demokratiequalität darstellen würde, besteht bei den ungültigen Stimmen die Möglichkeit, diese über eine noch bessere Informierung der Bevölkerung zumindest mittelfristig zu verringern. Ob das neue Wahlrecht daher, wie von Patrick Horst beschrieben, auf absehbare Zeit eine „Reformbaustelle“ bleiben wird, ist fraglich. ${ }^{31}$ Immerhin bewertet sowohl eine Mehrheit der Wähler als auch eine Mehrheit der Nichtwähler das Wahlrecht trotz seiner Komplexität - insgesamt positiv im Vergleich zu dem sehr viel einfacheren Wahlrecht von 2004. Die Akzeptanz des Wahlrechts ist also prinzipiell gegeben. Abzusehen ist daher von Vorschlägen, die erneut eine umfassende Reform fordern. Stattdessen sollte dem neuen Wahlrecht die Chance gegeben werden, sich bei einem noch besseren Informationsstand der Wähler in nahezu unveränderter Weise in weiteren Wahlgängen zu bewähren, denn fortdauernde Veränderungen können auch eine abschreckende Wirkung auf die Wähler ausüben.

Übrig bleiben damit letztlich nur noch kleinere Probleme des Wahlrechts, bei denen die Möglichkeit einer konsensualen Lösung eher gegeben ist. Zu denken ist insbesondere an die niedrigen Stimmzahlen, die manche Kandidaten auf den Landeslisten benötigten, um in die Bürgerschaft einzuziehen. Hier wäre in der Tat die Einführung eines Quorums sinnvoll. Ebenso kann auf den Landeslisten über die Reduzierung der Listenplätze nachgedacht werden, da das Personalangebot von 60 Kandidaten doch übertrieben erscheint und eher zur Unübersichtlichkeit beiträgt, als dass ein Mehrwert für die Demokratie zu erkennen wäre. Diese Nachteile sind offensichtlich zu erkennen; Verbesserungen könnten daher parteiübergreifend beschlossen und auch von der Bürgerinitiative „Mehr Demokratie“ akzeptiert werden. Unabhängig von solchen kleinen Reformvorschlägen sollte jedoch bei der Diskussion um das neue Wahlrecht bedacht werden, dass es in erster Linie die Parteien sind, die durch ihre Programme, Kandidaten und den fairen Wettstreit für die Qualität der parlamentarischen Demokratie Sorge zu tragen haben. Mögliche Probleme dem neuen Wahlrecht zuzuschreiben, verengt fälschlicherweise den Blick auf einen Nebenaspekt und ist einer Lösung der Probleme nicht zuträglich.

31 Vgl. Patrick Horst, a.a.O. (Fn. 1), S. 723. 